\title{
Article
}

\section{Fidelity to a motivational interviewing intervention for those with post-stroke aphasia: A small scale feasibility study}

Holland, Emma-Joy, Watkins, Caroline Leigh, Boaden, Elizabeth and Lightbody, Catherine Elizabeth

Available at http://clok.uclan.ac.uk/20387/

Holland, Emma-Joy ORCID: 0000-0003-3029-7573, Watkins, Caroline Leigh ORCID: 0000-0002-9403-3772, Boaden, Elizabeth ORCID: 0000-0002-46476392 and Lightbody, Catherine Elizabeth ORCID: 0000-0001-5016-3471

(2018) Fidelity to a motivational interviewing intervention for those with poststroke aphasia: A small scale feasibility study. Topics in Stroke Rehabilitation, 25 (1). pp. 54-60. ISSN 1074-9357

It is advisable to refer to the publisher's version if you intend to cite from the work. http://dx.doi.org/10.1080/10749357.2017.1376916

For more information about UCLan's research in this area go to

http://www.uclan.ac.uk/researchgroups/ and search for <name of research Group>.

For information about Research generally at UCLan please go to http://www.uclan.ac.uk/research/

All outputs in CLoK are protected by Intellectual Property Rights law, including Copyright law. Copyright, IPR and Moral Rights for the works on this site are retained by the individual authors and/or other copyright owners. Terms and conditions for use of this material are defined in the policies page. 
1 Fidelity to a motivational interviewing intervention for those with post-

2 stroke aphasia: A small scale feasibility study

3

\section{Abstract}

5

6

Methods: In a small-scale feasibility study, consecutive patients admitted to an acute stroke ward were screened for eligibility. People with moderate to severe aphasia were eligible. Those consenting received an intervention consisting of up to eight $\mathrm{Ml}$ sessions delivered twice per week over four weeks. Sessions were modified using aids and adaptations for aphasia. Session quality was measured using the Motivational Interviewing Skills Code (MISC) to assess MI fidelity.

Results: Three consenting patients identified early post-stroke took part; one male and two females ages ranging between 40s to 80 s. Participants attended between five to eight $\mathrm{MI}$ sessions over four weeks. Aids and adaptations included visual cues, rating scales and modified reflections incorporating verbal and non-verbal behaviours. Sessions were tailored to individual participant need. Threshold MISC ratings could be achieved for all participants however, ratings were reduced when aids and adaptations were not used.

Discussion: This small-scale feasibility study suggests that it is feasible to adapt MI for people with moderate to severe post-stroke aphasia. These findings merit further exploration of adapted MI as an intervention for this patient group.

Key words: Stroke; Stroke survivors; Aphasia; Motivational interviewing; Feasibility studies. 
26 Stroke recovery requires emotional adjustment, and depression post-stroke is common, with a third of stroke survivors experiencing symptoms [1]. Post-stroke depression is an independent predictor of recovery and quality of life [2], therefore early prevention and treatment is vital. A review of interventions for preventing depression post-stroke found psychotherapeutic interventions to be more effective than pharmacological [3]. However, talk-based therapies may need adjusting for those post-stroke, who can suffer from cognitive or communication difficulties. Whilst a third of stroke survivors will experience communication difficulties [4] impeding participation in talk-based therapies, little guidance exists on delivery in people with aphasia. We need to understand how to adapt talk-based therapies in the presence of aphasia.

Motivational interviewing (MI) is a talk-based therapy that has been shown to benefit patient mood post-stroke [5]. MI principles were used to increase awareness of the importance of changing what people make of their situation (adjustment), through amplifying the discrepancy between their current concerns and future goals or personal values and current approaches to addressing them. By reducing ambivalence and strengthening motivation, therapists explore a person's reason for changing what they make of their situation. Confidence to adjust to their current state is reinforced through supporting self-efficacy, enabling the person to develop motivation, and creating readiness to adjust [6]. Specific MI consistent techniques allow delivery of these principles; asking open questions, reflecting statements, providing affirmations and summarising. MI inconsistent techniques include, confronting people or giving advice without permission. Whilst data from this study seemed to indicate a particular benefit for those with mild aphasia [5], it is unclear whether it is possible in those with moderate to severe aphasia. Furthermore, if the delivery of MI needs adjustments, it is not known whether an adapted form of MI can maintain core MI principles. MI has previously been adapted for other populations including learning disabilities [7]. A pilot study of people with learning disabilities and alcohol dependency who experienced communication difficulties, incorporated adaptations including reading aloud materials for those unable to read, and 
51 providing regular summaries of topics discussed. Visual analogue scales were used to rate the

52 importance of, or confidence in, a topic; an MI strategy usually discussed verbally. These aids and adaptations improved patients' understanding. While the delivery of MI with people with learning difficulties may differ to those experiencing post-stroke aphasia, some adaptations used may be useful post-stroke.

More widely, methods to facilitate the participation of people with communication difficulties in research has involved using words and/or pictures to visualise information [8], incorporating nonverbal behaviour, simplifying questions, supporting comprehension and expression, checking that participants have been understood correctly [9] and training communication partners [10]. Aids and adaptations can facilitate communication for people who may struggle verbally, making it possible for people with aphasia to participate in a talk-based therapy. To date, despite data suggesting a potential benefit of $\mathrm{MI}$ to people with aphasia post-stroke [5], no study has explored the feasibility of how to adapt $\mathrm{MI}$ for this group.

A systematic review of interventions to prevent and treat depression in those with post-stroke aphasia [11] found various interventions that could be considered for those with sub-threshold to mild depression. However, the review highlighted a need to strengthen the evidence base and adapt preventative and treatment interventions. In order to do this, trials must be reported in a way that allows study replication and comparisons [11, 12]. However when adapting talk-based therapies, it is important that the adapted intervention maintains fidelity to the core principles of the therapy. Treatment fidelity builds confidence that changes to the dependent variable are attributable to the independent variable, in this case the talk-based therapy. This can be measured using various factors (design, training, delivery, receipt and enactment) [12]. severe post-stroke aphasia. This was achieved through two objectives, i) documenting the aids and 
adaptations utilised, and ii) observing how the utilisation of aids and adaptations affects MI fidelity. The study did not explore the impact of MI on mood outcomes.

\section{Methods}

Ethical approval was granted (August 2012) from National Research Ethics Service: North-West Preston. The feasibility study was nested within a larger study, performed on an acute stroke unit (ASU). The larger study explored delivering $\mathrm{MI}$ in patients with no or mild communication difficulties post-stroke and not those with moderate to severe aphasia. Consecutive people with suspected stroke admitted to the ASU May-December 2013 were screened for eligibility. People were eligible if: aged 18 or over; diagnosis of stroke; medically stable; moderate to severe aphasia based on the Communication Observational Assessment Tool, (COAT) [13], capable of consent; and living within the hospital catchment. Patients were ineligible if they were receiving psychological input (receiving treatment from a psychology professional) or had no verbal expression. Patients with mild communication difficulties were excluded but would have been eligible for the larger study.

Formal screening for communication ability was not standard practice on the ASU. To screen for the study, an observational tool was required which was i) non-invasive to the patient, ii) for clinical team use, and iii) able to categorise communication ability through routine observations. The COAT was used (See Appendix 1), based on Speech and Language Therapist (SLT) guidance allowing clinical staff (therapy or nursing) to screen to rate communication using five levels (none/ mild/ moderate/ moderately severe/ severe communication difficulties).

Purposive sampling was utilised to recruit patients with a range of communication abilities. We aimed to select 6-12 people across the three communication levels (Severe/ Moderately severe) Moderate), with equal numbers from each. People meeting eligibility criteria were approached by the stroke research nurse or research assistant. Aphasia-friendly study information and consent 
forms (Appendices 2 and 3) were provided, based on guidance $[14,15]$. Consent was taken by the stroke research nurse, with written informed consent provided, or witnessed consent for those unable to write.

Participant demographic and stroke details were recorded. Baseline measures of functional dependence (Barthel, [16]), communication and mood were completed. Communication was assessed using the Frenchay Aphasia Screening Test (FAST, [17]) and the Comprehensive Aphasia Test (CAT, [18]), the results of which guided the choice of aids and adaptations to tailor communication and MI delivery. Mood was measured using two participant self-report tools, the DISCS [19] and Yale [20] with a score of 2 or more and 1 respectively indicating low mood. Where possible, carer-rated mood measures were administered using the Stroke Aphasic Depression Questionnaire-10 [21] and Signs of Depression Scale [22], with cut-points of 14 and 2 respectively indicating low mood.

Intervention design

Participants received up to eight sessions of MI, two half-hour sessions per week for four weeks.

Session duration and frequency were adapted from the four one-hour sessions in the original trial of $\mathrm{MI}$ in stroke [5] to lessen the cognitive demand and fatigue from engaging in MI. Sessions were video and audio-recorded, allowing therapists to later reflect on the session, prepare for the next session, and monitor consistency of technique. MI sessions were delivered by the same therapist, in hospital or at home according to participant choice post-discharge. Post-intervention, participants received usual care.

\section{MI therapists}

Three Therapy Assistants (TAs) from the ASU multi-disciplinary team received training covering: stroke foundations, core research principles, theoretical background to $\mathrm{MI}$ and the psychological mechanisms that effect change, and practical MI training, delivered by MI therapists from the original MI post-stroke trial [5], who also provided supervision.. The training lasted one day per week for nine weeks, including independent learning sessions. This was followed by a minimum of ten 
practice MI sessions with volunteers. Therapists delivered MI with patients with no or mild communication difficulties post-stroke, until confidence and threshold competency were achieved, assessed with the MI Treatment Integrity (MITI) Code [23]. Therapists were provided with an intervention manual, allowing them to monitor their delivery and increase the likelihood that the intervention was delivered as intended.

\section{Aids and adaptations}

133 Aids were physical prompts used to facilitate conversation, whilst adaptations were alterations to the delivery of spoken information. The aids utilised included a communication framework Talking Mats ${ }^{\circledR}$ [24] a simple low-tech method of facilitating communication; a set of cards each with a written word and a corresponding picture. Nine category cards provide a starter topic of conversation (domestic life, relationships, work and education, leisure, learning and thinking, ways of coping, communication, mobility, and self-care). For each category, an accompanying set of cards allows further exploration of each topic. Cards are used (thumbs up, thumbs down, unsure) to indicate response. People may include their own cards (e.g. picture of an activity or relative). The "ways of coping" cards reflecting different emotions, were used to respond to other category cards.

142 Cards are moved around the mat to express thoughts on a topic. While Talking Mats ${ }^{\circledR}$ may not be suitable for all participants; it has previously been used successfully in people with post-stroke aphasia [25], and was a useful resource to initiate conversations. Ahead of MI sessions, a single Talking Mats ${ }^{\circledR}$ training session was provided by the researcher; however tailoring this to patient needs was guided by the SLT.

147 A second aid utilised was the visual rating scale (VRS); a vertical scale where participants rated a response from 0-10. This was used to establish the level of importance or confidence around an issue. Finally, a photo-book was used as a conversation starter, and pen and paper were available for participants or therapists to use as necessary. 
152 Data analysis

153 Video and audio footage were uploaded and synchronised in NVivo 10. Data were analysed to document the use of aids and adaptations, incorporating verbal and non-verbal information. Video footage was annotated and audio data was transcribed and coded. The MI Skills Code (MISC, Version 2.1, [26]) was used instead of the MITI to evaluate therapist MI competence and fidelity (MI consistency), participant behaviours (patient engagement), and the interaction between the two (therapist and patient collaboration), thus offering a more detailed analysis than the MITI as the latter two are not included in the MITI.

The MISC was developed to analyse specific therapist behaviours, evaluating therapist adherence to MI principles (fidelity). Two aspects of MI delivery are assessed: Global ratings and individual utterances. Global ratings take a holistic view of MI sessions, establishing more broadly whether there is adherence to the 'spirit' of MI. MISC global ratings reflect MI spirit, empathy, acceptance, egalitarianism, genuineness and warmth. Scores range from 0-7 (higher scores indicating greater adherence) and provide an overview of MI principles demonstrated in each session. Global ratings allow evaluation of i) the therapist's performance, ii) the person's involvement, and iii) the collaboration between therapist and person. This indicates the therapeutic alliance established.

Therapist's individual utterances are used to calculate the proportion of MI consistent responses. A second researcher independently coded half of the sessions for global MISC ratings to validate interpretation. To calculate the overall proportion of MI consistent utterances per session, each therapist utterance was coded as $\mathrm{MI}$ consistent (MICO) or MI inconsistent (MIIN). Therapist competence is established following MISC guidelines [26] which recommends minimum therapist

173 proficiency levels to achieve 'expert' or 'threshold' scores (for experienced or novice therapists

174 respectively). To reach threshold competency, therapists must achieve over five in global ratings and 

utterances .

179 Between May-December 2013, 201 patients with suspected stroke were screened for eligibility. Eleven were eligible and three consented to participate. A summary of screening and recruitment data is presented in Figure 1.

Due to staff attrition, one therapist was the sole provider of MI to the study. The therapist divided her time between her role as MI therapist and therapy assistant supporting SLT and dietetics stroke teams. The therapist had experience of working with people with aphasia utilising aspects of supported conversation, however formal communication training was not provided.

Demographic information and baseline measures

Demographic information for the three participants is presented in Table 1. At baseline, participants varied in level of aphasia, with participant 1 scoring considerably lower than other participants 2 on all communication measures. Participants differed in functional ability, level of social support

194 (separated/married/widow), and post-stroke role change (working/social/family). No participant screened as having low mood, as seen in Table 2. 
Details of the MI intervention received are shown in Table 3. Participants began MI within 2-12 weeks post-stroke, attending between $5-7$ sessions.

Insert Table 3 here

\section{Aids and adaptations utilised in MI sessions}

Aids and adaptations used to facilitate communication, incorporating both verbal and non-verbal information were identified. Talking Mats ${ }^{\circledR}$ was particularly useful for enabling open questions and, through moving the appropriate card on to the mat, the participant was able to respond without relying on verbal or written communication alone. Talking Mats ${ }^{\circledR}$ was introduced to all participants, however only the participant with severe aphasia utilised this aid frequently. Talking Mats ${ }^{\circledR}$ cards were supplemented with pictures relevant to the participant in order to illustrate an idea, for example, using a picture of a care home the participant had recently visited.

The VRS, although initially intended for use in establishing the participant's level of importance or confidence of an issue was also employed by participants to respond to open questions. For example, to demonstrate how they felt about staying in hospital (low for dislike, higher indicating positivity).

Each participants' photo-book showed key aspects of their life, including family, pets, or holidays. The photo-book acted as a conversation starter for participant 1 in particular (severe aphasia), prompting discussion of home life and family. The participant with moderately severe aphasia used an aid of pen and paper, providing her with multiple routes to communicate.

SLT guidance included supportive conversation techniques, such as employing a slow pace of conversation, and allowing adequate time for participants' responses. The therapist also used aids to enhance the patient's understanding, including pointing to pictures or words that were being discussed to reinforce the message such as writing key words as they are discussed. Gesture was 
used by both therapist and participants, providing multiple methods to convey the same information. MI adaptations involved using increased reflections, including reflections of non-verbal information e.g. information conveyed through gesture. Summaries allowed participants to maintain focus on the conversation whilst simultaneously providing an opportunity for the therapist to ensure they had understood the participant. Table 4 highlights the MI strategies used.

$$
\text { >>Insert Table } 4 \text { here }<<
$$

\section{The impact of adaptations on MI fidelity}

A second researcher independently coded half of the sessions for global MISC ratings to validate interpretation. Full agreement of ratings or a one-point difference was achieved for $93 \%$. A twopoint difference occurred in $7 \%$ of ratings, and each was discussed until a consensus was reached.

Therapist levels of MI fidelity varied across sessions from sub-threshold to expert level. This variation in MI fidelity was most prominent in sessions delivered with participant 1, who had severe aphasia.

A higher level of MI fidelity was applied with participants 2 and 3; with most sessions reaching expert level.

The MI ratings are displayed in Table 5. It was expected that the therapist should reach a minimum of threshold level (over five in global ratings / 80\% MICO utterances) however for participant 1 (severe aphasia), therapist MI ratings varied from below threshold to expert level.

Participant 2 (moderately severe aphasia) therapist MISC ratings were good, with all sessions reaching threshold level and many sessions reaching expert level (over six for global ratings / 90\% MICO utterances). Participant MISC ratings reached expert level for all sessions except one session which achieved threshold level indicating strong engagement.

Participant 3 (moderate aphasia) therapist MISC ratings for participant were also good, with the therapist reaching threshold level in all sessions, and some sessions reaching expert levels. All 
patient MISC ratings reached expert level, indicating both therapist and participant were positively engaged with a strong therapeutic alliance.

To draw attention to the potential impact of aids and adaptations on the MI content, a summary of sessions with participant 1 (severe aphasia) is shown in Table 6.

MI has previously been used to prevent depression post-stroke [5]; however, this is the first study to provide support for the feasibility of delivering $\mathrm{MI}$ adapted for those with moderate to severe poststroke aphasia. While involving only a small number of participants, this study has demonstrated that when MI sessions were adapted to meet the needs of those with aphasia, MI principles and fidelity can be achieved. The MI therapist was able to reach threshold levels of MI fidelity, which was demonstrated through both MI consistent utterances and global ratings. Expert levels were achieved in some sessions, as is highlighted in Tables 4 and 5.

The therapist was able to maintain high levels of MI fidelity with participants with less severe aphasia (participants 2 and 3), however, struggled to maintain this level across sessions with participant 1 (severe aphasia). This is evident when comparing individual sessions for participant 1 (Table 5). Session 3 is the highest rated for MI consistency and spirit. The visual rating scale (VRS) was used 14 times, which may have facilitated 17 open questions. Closed questions were used 49 times; however, these are often required for people unable to provide more in-depth verbal responses. Therapist and participant MISC global ratings and overall MI fidelity both reached expert level, and $\mathrm{MI}$ inconsistent responses are low $(\mathrm{n}=3)$ indicating a successful session. In contrast, session 5 has a sub-threshold level of MI. The VRS was used five times, with only 2 open questions 
facilitated, and 131 closed questions used. Ratings for participant engagement and collaboration are low, with a higher number of $\mathrm{MI}$ inconsistent responses ( $n=17)$.

271 Aids and adaptations may have facilitated MI techniques, such as open questions and reflections,

272 and consequently the delivery of MI. When aids and adaptations were not used, participants were

273 limited in the information they could communicate. The therapist's ability to tailor sessions for participant 1 (severe aphasia) appeared to impact on MI fidelity as well as participant engagement.

However, it may not be that the use of aids and adaptations were responsible for the change in the ability to maintain MI principles. Other factors, including changes in circumstance experienced may have impacted on sessions. The participants experienced varying degrees of life changes post-stroke, including level of physical disability, social support, role change, and consequently had different issues to adjust to. Participant 1, who had multiple significant changes following the stroke, may have been more challenging for the therapist to engage with, than those with fewer or less significant issues to adjust to.

Previous MI trials were often limited by their lack of documentation to explain what intervention was delivered [27], reducing validity and replicability. In order to adequately demonstrate treatment fidelity $[12,27,28]$ in this study, a range of issues were considered and documented, including: session number, duration and content; therapist background, training and support; MI delivery and adherence to MI principles. The accurate reporting of delivering adapted MI may be particularly important to ensure transparency of what intervention has truly been delivered. Failure to do so, particularly in MI trials, has been highlighted as problematic [29].

The small number of participants limited the study, restricting our understanding of the impact of adaptations and a more informative analysis may have been possible with participants with a broader range of communication difficulties. A larger scale study may wish to investigate this 

further, and may consider using multiple therapists to deliver MI to further our knowledge of the impact of the therapist in this complex relationship.

294 The feasibility study indicates that the delivery of MI to those with post-stroke aphasia has potential

295 for future development. This study has implications for talk-based therapies post-stroke, in

296 particular for those who may struggle to engage in standard talk-based therapies. Adapted MI could

297 offer a form of psychological support that is not currently delivered.

298

299 Conflict of interest

$300 \quad$ None declared 
1. Hackett, M.L. and K. Pickles, Part I: Frequency of Depression after Stroke: An Updated Systematic Review and Meta-Analysis of Observational Studies. International Journal of Stroke. 2014; 9(8): 1017-1025.

2. Donnellan C, Hickey A, Hevey D, and O'Neill D. Effect of mood symptoms on recovery one year after stroke. Int J Geriatr Psychiatry. 2010; 25(12): 1288-1295.

3. Hackett ML, Anderson CS, House A, Halteh C. Interventions for preventing depression after stroke. Cochrane Database of Systematic Reviews. 2008;(3).

4. Kauhanen ML, Korpelainen JT, Hiltunen, P, et al. Aphasia, depression, and non-verbal cognitive impairment in ischaemic stroke. Cerebrovasc Dis. 2000; 10(6): 455-461.

5. Watkins $\mathrm{CL}$, Auton $\mathrm{MF}$, Deans $\mathrm{CF}$, et al. Motivational interviewing early after acute stroke: a randomized, controlled trial. Stroke. 2007; 38(3): 1004-1009.

6. Miller $W$ and Rollnick R. The effectiveness and ineffectiveness of complex behavioral interventions: Impact of treatment fidelity. Contemp Clin Trials. 2014; 37: 234-241.

7. Mendel E and Hipkins J. Motivating learning disabled offenders with alcohol-related problems: a pilot study. Brit J Learn Disabil. 2002; 30(4): 153-158.

8. Pearl G and Wade R. Facilitating the Involvement of People with Aphasia in Stroke Research by Developing Communicatively Accessible Research Resources. Top Lang Disorders. 2017; 37(1): 67-84.

9. Dalemans R, Wade DT, van den Heuvel WJ, and de Witte LP. Facilitating the participation of people with aphasia in research: a description of strategies. Clin Rehabil. 2009; 23(10): 948959.

10. Simmons-Mackie N, Raymer A, Cherney L. Communication Partner Training in Aphasia: An Updated Systematic Review. Arch Phys Med Rehabil. 2016; 97: 2202-2221.

11. Baker C, Worrall L, Rose M, Hudson K, Ryan B, and O'Byrne L. A systematic review of rehabilitation interventions to prevent and treat depression in post-stroke aphasia. Disabil Rehab. 2017. http://dx.doi.org/10.1080/09638288.2017.1315181 
12. Borrelli B, Sepinwall D, Ernst D, Bellg AJ, Czajkowski S, Breger R, DeFrancesco C, Levesque C, Sharp DL, Ogedegbe G, Resnik B and Orwig D. A new tool to assess treatment fidelity and evaluation of treatment fidelity across 10 years of health behavior research. J Consult Clin Psychol. 2005;73(5):852-60.

13. Holland EJ. The feasibility of delivering motivational interviewing those with communication difficulties following a stroke. Unpublished PhD thesis. University of Central Lancashire. 2009.

14. Engaging with people who have aphasia. A set of resources for stroke researchers. National Institute for Health Research. Clinical Research Stroke Network (NIHR CRN: Stroke). 2014.

15. Rose TA, Worrall L, Hickson LM, and Hoffman TC. Aphasia friendly written health information: content and design characteristics. Int J Speech Lang Pathol. 2011; Aug;13(4):335-47. doi: 10.3109/17549507.2011.560396.

16. Wade DT and Colin C. The Barthel ADL Index: a standard measure of physical disability? Int disabil Stud. 1988; 10(2): 64-7.

17. Enderby PM, Wood VA, Wade DT, and Hewer RL. The Frenchay Aphasia Screening Test: a short, simple test for aphasia appropriate for non-specialists. Int Rehabil Med 1987; 8(4): 166-170.

18. Swinburn K, Porter G, and Howard D. CAT: comprehensive aphasia test. Psychology Press: Hove; 2004.

19. Turner-Stokes L, Kalmus M, Hirani D, and Clegg F. The Depression Intensity Scale Circles (DISCS): A first evaluation of a simple assessment tool for depression in the context of brain injury. J Neurol, Neurosurg Psychiatry. 2005; 76(9): 1273-1278.

20. Mahoney J, Drinka TJ, Abler R, Gunter-Hunt G, Matthews C, Gravenstein S and Carnes M. Screening for depression: single question versus GDS. J Am Geriatr Soc. 1994; 42(9): 10061008. 
21. Sutcliffe $L$ and Lincoln $N$. The assessment of depression in aphasic stroke patients: the development of the Stroke Aphasic Depression Questionnaire. Clin Rehabil. 1998; 12(6): 506513.

22. Hammond MF, O'Keeffe ST, and Barer DH. Development and validation of a brief observerrated screening scale for depression in elderly medical patients. Age Ageing. 2000; 29: 51115.

23. Moyers T, Martin T, Manuel JK, and Miller WR. The Motivational Interviewing Treatment Integrity (MITI) Code: Version 2.0. University of New Mexico: Center on Alcoholism, Substance Abuse and Addictions; 2005.

24. Murphy J. Talking Mats: speech and language research in practice. Speech and Language Therapy in Practice. Autumn 1998: 11-14.

25. Murphy J. Enabling people with aphasia to discuss quality of life. BJTR. 2000; 7(11): 454-458.

26. Miller WR, Moyers TB, Ernst D, Amrhein P. Manual for the motivational interviewing skill code (MISC) Version 2.1. 2008. Retrieved from http://casaa.unm.edu/download/misc.pdf.

27. Rollnick S. Comments on Dunn et al's "The use of brief interventions adapted from motivational interviewing across behavioral domains: a systematic review". Enthusiasm, quick fixes and premature controlled trials. Addiction. 2001:96(12):1769-1770.

28. Hinckley JJ, and Douglas NF. Treatment fidelity: its importance and reported frequency in aphasia treatment studies. Am J Speech Lang Pathol. 2013; 22: S279-S284

29. Miller WR and Rollnick S. The effectiveness and ineffectiveness of complex behavioural interventions: Impact of treatment fidelity. Contemp Clin Trials. 2014; 37: 234-241. 
Table 1. Demographic information and baseline scores of communication, cognition and functional dependence

\section{Participant $1 \quad$ Participant $2 \quad$ Participant 3}

\begin{tabular}{llll}
\hline Sex & Male & Female & Female \\
Age & 44 & 65 & 87
\end{tabular}

\section{Screening measure}

Level of communication difficulty (COAT) Severe Moderately Moderate

Severe

Communication: FAST (max. 30)

1

23

16

CAT expression: Repetition

0/50, 0/74

$27 / 50,54 / 74$

$20 / 50,38 / 74$

Naming

$0 / 29,0 / 58$

$16 / 29,29 / 58$

$13 / 29,24 / 58$

Reading

$0 / 35,0 / 70$

$26 / 35,56 / 70$

$27 / 35,54 / 70$

Written Language

$0 / 76$

$54 / 76$

$49 / 76$

CAT Comprehension of written language

$10 / 62$

$52 / 62$

$46 / 62$

Comprehension of spoken language

$15 / 66$

$52 / 66$

$56 / 66$

CAT Cognitive screen

$9 / 38$

$37 / 38$

$30 / 38$

Functional dependence: Barthel $(\max 20)$

4

20

19

*COAT= Communication Observational Assessment Tool, FAST=Frenchay Aphasia Screening Test, CAT=Comprehensive Aphasia Test, 
Table 2. Baseline mood scores

\begin{tabular}{lccc}
\hline Mood tool & Participant 1 & Participant 2 & Participant 3 \\
\hline Yale single-item & 0 & 0 & 0 \\
DISCs (max. 5) & 0 & 1 & 1 \\
SODS & Not available & 1 & 1 \\
SADQ-10 & Not available & 8 & 12
\end{tabular}

Yale single-item (cut-off 1), DISCs=Depression Intensity Scale Circles (cut-off 2), SODS=Signs Of Depression Scale (cut-off 2)), SADQ-10=Stroke Aphasic Depression Questionnaire-10 (cut-off 14). 
Participant 1 Participant $2 \quad$ Participant 3

Number of $\mathrm{Ml}$ sessions received

Time from stroke to first MI session (weeks)

Duration of intervention (weeks)

Length of sessions in minutes Mean (range)

\section{5}

12

7

5

2.5

4

5

$21(16-30) \quad 23(18-29) \quad 29(13-40)$ 
1 Table 4. Session communication strategy and MISC code ratings

\begin{tabular}{ccccccccc}
\hline Participant & $\begin{array}{c}\text { Total no. } \\
\text { of } \\
\text { sessions }\end{array}$ & $\begin{array}{c}\text { Use of VRS } \\
\text { Median (range) }\end{array}$ & $\begin{array}{c}\text { Open questions: } \\
\text { Median (range) }\end{array}$ & $\begin{array}{c}\text { Closed } \\
\text { questions: } \\
\text { Median (range) }\end{array}$ & $\begin{array}{c}\text { Summaries: } \\
\text { Median (range) }\end{array}$ & $\begin{array}{c}\text { Reflections: } \\
\text { Median (range) }\end{array}$ & $\begin{array}{c}\text { Affirmations: } \\
\text { Median (range) }\end{array}$ & $\begin{array}{c}\text { Overall MI } \\
\text { consistency** } \\
(\%)\end{array}$ \\
range & $71-95$ \\
\hline 1 & 5 & $5(2-14)$ & $8(2-17)$ & $72(49-131)$ & $1(0-7)$ & $19(10-28)$ & $6(2-12)$ \\
3 & 8 & $1.5(0-6)$ & $4.5(3-12)$ & $26(18-78)$ & $2(0-7)$ & $21(10-26)$ & $2(0-8)$ & $93-100$ \\
3 & 7 & $1(0-4)$ & $2.5(0-8)$ & $21.5(6-28)$ & $2.5(1-9)$ & $10(3-14)$ & $2.5(0-8)$ & $88-100$
\end{tabular}

** Ml consistent utterances scored 0-100\%, with over 80\% reaching threshold level and over $90 \%$ for expert level. 
4 Table 5. Patient MISC and overall MI consistency ratings

\begin{tabular}{|c|c|c|c|c|c|}
\hline \multirow[t]{2}{*}{ Participant } & \multirow[t]{2}{*}{$\begin{array}{l}\text { Total no. of } \\
\text { sessions }\end{array}$} & \multirow[t]{2}{*}{$\begin{array}{l}\text { Therapist MI spirit rating*: } \\
\text { Median (range) }\end{array}$} & $\begin{array}{c}\text { Patient } \\
\text { engagement rating*: }\end{array}$ & \multirow{2}{*}{$\begin{array}{c}\text { Therapist \& Patient } \\
\text { collaboration rating*: } \\
\text { Median (range) }\end{array}$} & \multirow[t]{2}{*}{$\begin{array}{c}\text { Overall MI consistency** }(\%) \\
\text { (range) }\end{array}$} \\
\hline & & & Median (range) & & \\
\hline 1 & 5 & $4(4-6)$ & $6(4-6)$ & $5(3-5)$ & $71-95$ \\
\hline 2 & 8 & $6(5-6)$ & $6(5-6)$ & $5(5-6)$ & $93-100$ \\
\hline 3 & 7 & $5.5(5-6)$ & $6(6-7)$ & $5.5(5-6)$ & $88-100$ \\
\hline
\end{tabular}

$5 \quad$ *Global ratings scored from 0-7, with scores over 5 reaching threshold level and over 6 for expert level

$6 \quad * *$ MI consistent utterances scored $0-100 \%$, with over $80 \%$ reaching threshold level and over $90 \%$ for expert level. 
Table 6. Summary of aids and adaptations used and MI content of sessions with a person with severe aphasia

\begin{tabular}{|c|c|c|c|c|c|c|c|c|c|c|c|}
\hline Sess. & $\begin{array}{c}\text { Use } \\
\text { of } \\
\text { VRS }\end{array}$ & $\begin{array}{c}\text { Open } \\
\text { questions }\end{array}$ & $\begin{array}{c}\text { Closed } \\
\text { questions }\end{array}$ & Reflections & Summaries & $\begin{array}{l}\text { Therapist } \\
\text { MI spirit } \\
\text { rating* }\end{array}$ & $\begin{array}{c}\text { Patient } \\
\text { engagement } \\
\text { rating* }\end{array}$ & $\begin{array}{c}\text { Therapist \& } \\
\text { patient } \\
\text { collaboration } \\
\text { rating* }\end{array}$ & $\begin{array}{c}\text { MI } \\
\text { inconsistent } \\
\text { responses } \\
* * \%\end{array}$ & $\begin{array}{c}\text { MI } \\
\text { consistent } \\
\text { responses } \\
* * \%\end{array}$ & $\begin{array}{c}\text { Overall MI } \\
\text { consistency } \\
* * \%\end{array}$ \\
\hline 1 & 3 & 8 & 72 & 19 & 7 & 4 & 5 & 4 & 5 & 38 & 88 \\
\hline 2 & 7 & 13 & 59 & 18 & 0 & 4 & 6 & 5 & 5 & 44 & 90 \\
\hline 3 & 14 & 17 & 49 & 25 & 1 & 6 & 6 & 5 & 3 & 52 & 95 \\
\hline 4 & 2 & 5 & 99 & 10 & 0 & 4 & 6 & 5 & 15 & 37 & 71 \\
\hline 5 & 5 & 2 & 131 & 28 & 4 & 4 & 4 & 3 & 17 & 44 & 72 \\
\hline
\end{tabular}

9 Sess. $=$ Sessions, VRS=Visual rating scale. ${ }^{*}$ Global ratings scored from $0-7$, with scores over 5 reaching threshold level and over 6 for expert level. ${ }^{* *} \mathrm{MI}$ consistent utterances 10 scored $0-100 \%$, with over $80 \%$ reaching threshold level and over $90 \%$ for expert level. 
13 Communication Observation Checklist (Please Tick One)

\begin{tabular}{|c|c|c|c|}
\hline WS2 & \begin{tabular}{|l} 
Please \\
Tick
\end{tabular} & & $\begin{array}{l}\text { Please } \\
\text { Tick }\end{array}$ \\
\hline Level 1: No Observed Difficulties & & $\begin{array}{l}\text { Level 2: Mild Communication } \\
\text { Problems } \\
\text {-reduced verbal expression and } \\
\text { fluency } \\
\text {-speaks in sentences } \\
\text {-may have occasional word } \\
\text { finding difficulties } \\
\text {-able to have a conversation } \\
\text {-engages in turn taking }\end{array}$ & \\
\hline WS3 & & & \\
\hline $\begin{array}{l}\text { Level 3: Moderate Aphasia } \\
\text {-may speak in phrases } \\
\text {-may be able to use longer sentences } \\
\text {-may have occasional word finding } \\
\text { difficulties } \\
\text {-sound substitution errors may occur }\end{array}$ & & $\begin{array}{l}\text { Level 4: Moderately Severe } \\
\text { Aphasia } \\
\text {-poor expression using only short } \\
\text { phrases or single words }\end{array}$ & \\
\hline $\begin{array}{l}\text { Level 5: Severe Aphasia } \\
\text {-unable to speak in phrases } \\
\text {-severe word finding difficulties } \\
\text {-reduced expression due to dysarthria only } \\
\text {-someone who relies purely on gesture or a } \\
\text { communication chart to communicate }\end{array}$ & & & \\
\hline
\end{tabular}




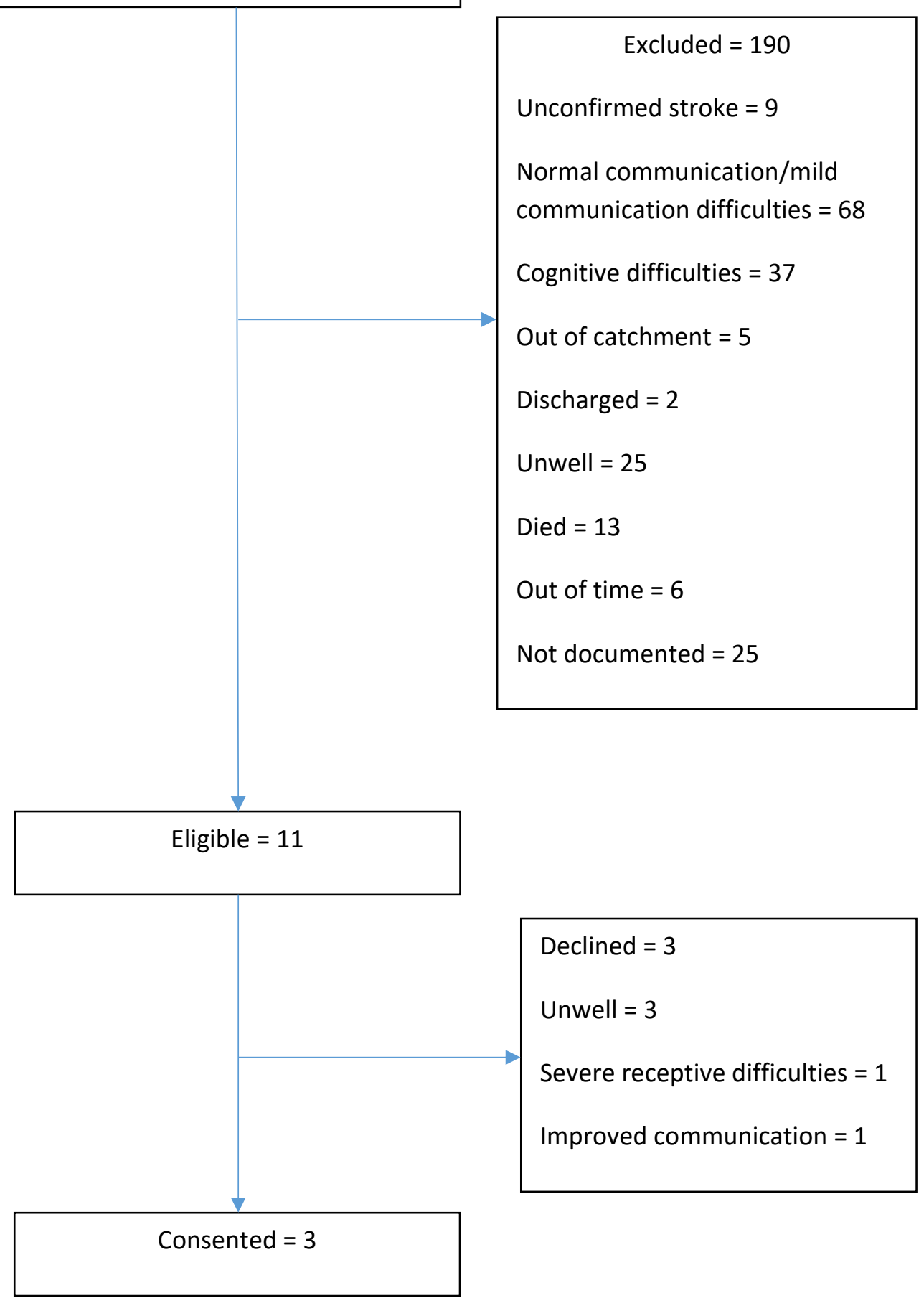

Figure 1: Screening and recruitment to the feasibility study 